Supporting Information for:

\title{
Long-range electrostatic colloidal interactions and specific ion effects in deep eutectic solvents
}

Adrian Sanchez-Fernandez, ${ }^{a *}$ Andrew J. Jackson, ${ }^{b, c}$ Sylvain F. Prévost, ${ }^{d}$ James J. Doutch, ${ }^{e}$ Karen J. Edler ${ }^{f}$ ${ }^{\mathrm{a} F o o d}$ Technology, Engineering and Nutrition, Lund University, Box 124, 22100 Lund, Sweden.

${ }^{b}$ European Spallation Source, Box 176, 22100 Lund, Sweden.

'Department of Physical Chemistry, Lund University, Lund, SE-221 00, Sweden.

'Institut Laue-Langevin, 71 Avenue des Martyrs, 38000, Grenoble, France.

eISIS Neutron and Muon Source, Science and Technology Facilities Council, Rutherford Appleton Laboratory, Didcot, OX11 0QX, UK.

fDepartment of Chemistry, University of Bath, Claverton Down, Bath, BA2 7AY, UK.

*E-mail: adrian.sanchez-fernandez@food.Ith.se 


\section{Synthesis and characterization of the counterion-exchanged surfactant}

The counterion-exchanged surfactants were prepared by exchanging the native chloride counterion from dodecyltrimethylammonium chloride $\left(\mathrm{C}_{12} \mathrm{TAC}\right.$, Sigma-Aldrich, $\left.>99 \%\right)$ using a modification of the procedure previously reported for the cationic surfactants. ${ }^{1}$ The ion exchange resin Amberlite IRN-78 hydroxide form (Sigma-Aldrich) was loaded into a chromatography column with a porous frit to retain the particle packing. The resin was initially washed using a $1 \mathrm{M} \mathrm{NaOH}$ solution and subsequently washed with abundant Milli-Q water to remove any excess base remanent in the column. Once the eluted solvent was neutral in $\mathrm{pH}$, the column was ready for the counterion exchange.

$\mathrm{C}_{12} \mathrm{TAC}$ was dissolved in milli-Q water ( $c a .800 \mathrm{mM}$ ) and loaded into the column at room temperature. The solution was subsequently pass through the column at least 5 times. The complete exchange was verified by the addition of a drop of silver nitrate solution to an aliquot of the surfactant solution. The exchange was completed when no $\mathrm{AgCl}$ precipitation could be observed, confirming the absence of any remanent chloride in the surfactant solution.

The resulting solution of dodecyltrimethylammonium hydroxide was neutralized to $\mathrm{pH} 7$ using the corresponding acid solutions ( $1 \mathrm{M}): \mathrm{HBr}, \mathrm{HNO}_{3}$, or $\mathrm{H}_{2} \mathrm{SO}_{4}$. The neutralized solution was freeze-dried to remove the water and the powder was recovered for purification. The surfactants were dissolved and triplerecrystallized using ethanol/dry diethyl ether. Any residual solvent was removed in vacuo. The purity of the surfactants was assessed by ${ }^{1} \mathrm{H}-\mathrm{NMR}$.

\section{Results from the SANS data analysis}

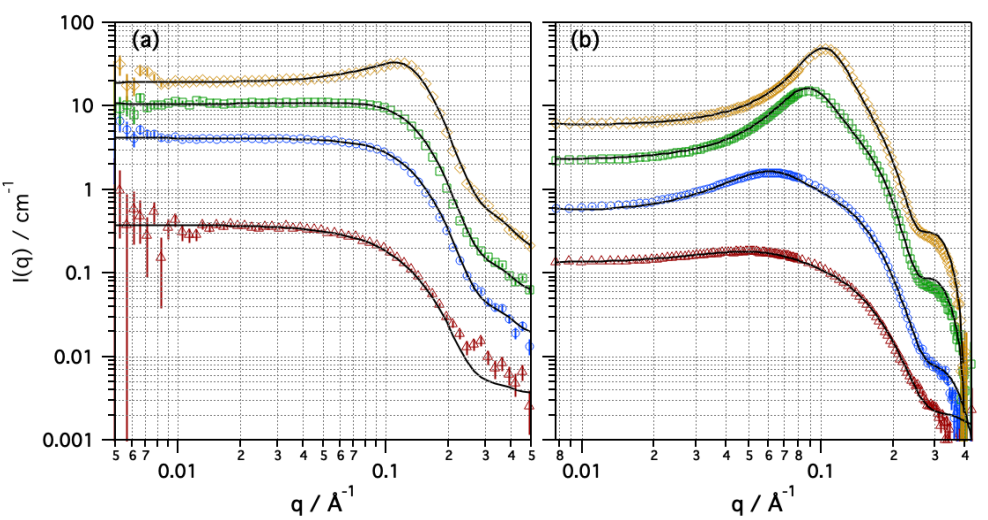

Figure S1 SANS data and best fits from different concentrations of h- $\mathrm{C}_{12}$ TAC micelles in (a) 1:2 d-ChCl:d-Glyc (78.8 mM$\triangle, 203 \mathrm{mM}-\bigcirc, 437 \mathrm{mM}-\square$, and $1070 \mathrm{mM}-\diamond$ ) and (b) $\mathrm{D}_{2} \mathrm{O}$ (39.4 mM- $\triangle$, $78.8 \mathrm{mM}-\bigcirc, 203 \mathrm{mM}-\square$, and $\left.368 \mathrm{mM}-\diamond\right)$. Data were modelled using the uniform ellipsoid $P(q)$ and RMSA S(q) implemented using the DA. Data and models were scaled for clarity. Where not seen, the error bars are within the markers.

Table S1 Parameters obtained from the analysis of the SANS data presented in Figure S1: equatorial radius $-r_{\text {eq, }}$, aspect ratio $-A R$, micelle volume fraction $-\phi$, $q$-position of the first peak in the $S(q)$ data $-q_{s m a x}, S(q)$ intensity at zero angle $\mathrm{S}(0)$, and Coulomb coupling factor $-\Gamma_{\mathrm{k}}$.

\begin{tabular}{|c|c|c|c|c|c|c|}
\hline$\left[\mathrm{C}_{12} \mathrm{TAC}\right] / \mathrm{mM}$ & $\mathrm{r}_{\text {eq }} / \AA$ & $\mathrm{AR}$ & $\phi_{p} / \times 10^{-2}$ & $\mathrm{q}_{\text {smax }} / \AA^{-1}$ & $\mathrm{~S}(0) / \mathrm{cm}^{-1}$ & $\Gamma_{\mathrm{k}}$ \\
\hline \multicolumn{7}{|c|}{$\mathrm{h}-\mathrm{C}_{12} \mathrm{TAC}$ in d-ChCl:d-Glyc } \\
\hline 78.8 & $14.3 \pm 0.3$ & $1.71 \pm 0.06$ & $0.35 \pm 0.03$ & $0.113 \pm 0.053$ & $0.942 \pm 0.075$ & $4.25 \times 10^{-31} \pm 1.01 \times 10^{-10}$ \\
\hline 203 & $14.3 \pm 0.3$ & $1.71 \pm 0.06$ & $2.79 \pm 0.04$ & $0.129 \pm 0.004$ & $0.677 \pm 0.027$ & $1.03 \times 10^{-13} \pm 9.28 \times 10^{-14}$ \\
\hline 437 & $14.3 \pm 0.3$ & $1.71 \pm 0.06$ & $4.87 \pm 0.05$ & $0.133 \pm 0.003$ & $0.499 \pm 0.010$ & $1.24 \times 10^{-10} \pm 1.64 \times 10^{-12}$ \\
\hline 1070 & $14.3 \pm 0.3$ & $1.71 \pm 0.06$ & $10.6 \pm 0.1$ & $0.143 \pm 0.002$ & $0.193 \pm 0.004$ & $8.62 \times 10^{-7} \pm 4.97 \times 10^{-9}$ \\
\hline \multicolumn{7}{|c|}{ h-C $12 \mathrm{TAC}$ in $\mathrm{D}_{2} \mathrm{O}$} \\
\hline 39.4 & $14.1 \pm 0.2$ & $1.49 \pm 0.06$ & $0.23 \pm 0.03$ & $0.069 \pm 0.002$ & $0.637 \pm 0.012$ & $3.74 \times 10^{-6} \pm 1.93 \times 10^{-8}$ \\
\hline 78.8 & $15.1 \pm 0.2$ & $1.45 \pm 0.06$ & $0.93 \pm 0.03$ & $0.073 \pm 0.002$ & $0.279 \pm 0.006$ & $1.01 \times 10^{-3} \pm 5.56 \times 10^{-5}$ \\
\hline 203 & $16.5 \pm 0.1$ & $1.33 \pm 0.04$ & $4.82 \pm 0.02$ & $0.099 \pm 0.001$ & $0.096 \pm 0.002$ & $4.8 \times 10^{-2} \pm 5.91 \times 10^{-4}$ \\
\hline 368 & $16.8 \pm 0.1$ & $1.31 \pm 0.04$ & $8.49 \pm 0.02$ & $0.115 \pm 0.001$ & $0.067 \pm 0.001$ & $1.32 \times 10^{-1} \pm 2.16 \times 10^{-4}$ \\
\hline
\end{tabular}



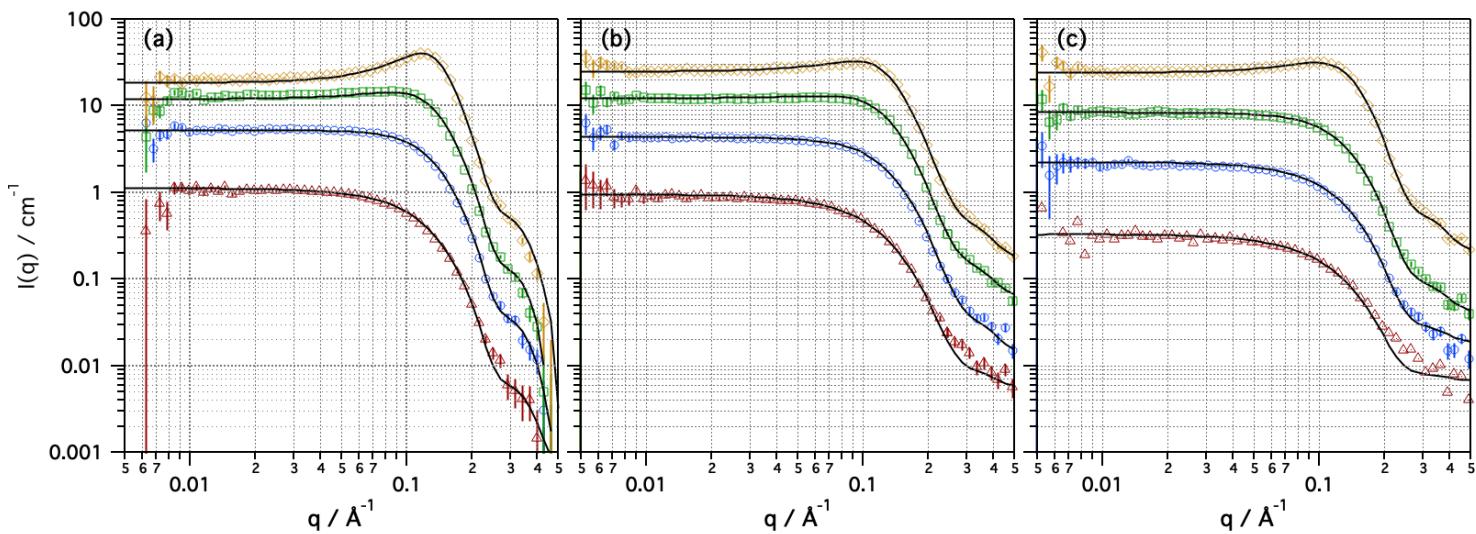

Figure S2 SANS data and best fits from (a) $h-C_{12} T A B$, (b) $h-C_{12} \mathrm{TANO}_{3}$, and (c) h-C $\mathrm{C}_{12} \mathrm{TA}\left(\mathrm{SO}_{4}\right)_{1 / 2}$ micelles in 1:2 d-ChCl:d-Glyc at different surfactant (average) concentrations (78.8 mM- $\triangle, 203 \mathrm{mM}-\bigcirc, 437 \mathrm{mM}-\square$, and $1070 \mathrm{mM}-\diamond)$. Data were modelled using the uniform ellipsoid $\mathrm{P}(\mathrm{q})$ and RMSA $\mathrm{S}(\mathrm{q})$ implemented using the DA. Data and models were scaled for clarity. Where not seen, the error bars are within the markers.

Table S2 Structural parameters obtained from the analysis of the SANS data presented in Figure S2.

\begin{tabular}{|c|c|c|c|c|c|c|}
\hline$\left[\mathrm{C}_{12} \mathrm{TA}^{+}\right] / \mathrm{mM}$ & $\mathrm{r}_{\text {eq }} / \AA$ & $\mathrm{AR}$ & $\phi_{p} / \times 10^{-2}$ & $\mathrm{q}_{\mathrm{smax}} / \AA^{-1}$ & $\mathrm{~S}(0) / \mathrm{cm}^{-1}$ & $\Gamma_{\mathrm{k}}$ \\
\hline \multicolumn{7}{|c|}{$\mathrm{h}-\mathrm{C}_{12} \mathrm{TAB}$ in d-ChCl:d-Glyc } \\
\hline 79.7 & $15.8 \pm 0.3$ & $1.38 \pm 0.08$ & $1.08 \pm 0.03$ & $0.092 \pm 0.073$ & $0.868 \pm 0.069$ & $1.38 \times 10^{-20} \pm 1.01 \times 10^{-8}$ \\
\hline 205 & $15.8 \pm 0.3$ & $1.38 \pm 0.08$ & $3.60 \pm 0.05$ & $0.125 \pm 0.008$ & $0.616 \pm 0.025$ & $1.54 \times 10^{-12} \pm 4.28 \times 10^{-20}$ \\
\hline 422 & $15.8 \pm 0.3$ & $1.38 \pm 0.08$ & $7.01 \pm 0.08$ & $0.136 \pm 0.006$ & $0.362 \pm 0.007$ & $6.22 \times 10^{-9} \pm 1.64 \times 10^{-15}$ \\
\hline 943 & $15.8 \pm 0.3$ & $1.38 \pm 0.08$ & $12.6 \pm 0.1$ & $0.146 \pm 0.002$ & $0.155 \pm 0.003$ & $4.77 \times 10^{-6} \pm 4.97 \times 10^{-9}$ \\
\hline \multicolumn{7}{|c|}{ h- $\mathrm{C}_{12} \mathrm{TANO} \mathrm{N}_{3}$ in d-ChCl:d-Glyc } \\
\hline 83.9 & $14.2 \pm 0.4$ & $1.84 \pm 0.11$ & $0.90 \pm 0.05$ & $0.114 \pm 0.092$ & $0.847 \pm 0.068$ & $3.43 \times 10^{-22} \pm 1.10 \times 10^{-10}$ \\
\hline 200 & $14.2 \pm 0.4$ & $1.84 \pm 0.11$ & $2.87 \pm 0.04$ & $0.115 \pm 0.011$ & $0.680 \pm 0.027$ & $7.42 \times 10^{-14} \pm 1.28 \times 10^{-16}$ \\
\hline 426 & $14.2 \pm 0.4$ & $1.84 \pm 0.11$ & $5.37 \pm 0.05$ & $0.132 \pm 0.007$ & $0.438 \pm 0.009$ & $1.54 \times 10^{-10} \pm 1.64 \times 10^{-11}$ \\
\hline 932 & $14.2 \pm 0.4$ & $1.84 \pm 0.11$ & $8.22 \pm 0.06$ & $0.136 \pm 0.003$ & $0.297 \pm 0.006$ & $1.93 \times 10^{-8} \pm 1.47 \times 10^{-9}$ \\
\hline \multicolumn{7}{|c|}{$\left.\mathrm{h}-\mathrm{C}_{12} \mathrm{TA}(\mathrm{SO})_{4}\right)_{1 / 2}$ in d-ChCl:d-Glyc } \\
\hline 86.0 & $15.1 \pm 0.2$ & $1.50 \pm 0.06$ & $0.29 \pm 0.07$ & $0.103 \pm 0.067$ & $0.969 \pm 0.077$ & $1.09 \times 10^{-33} \pm 1.11 \times 10^{-10}$ \\
\hline 205 & $15.1 \pm 0.2$ & $1.50 \pm 0.06$ & $1.13 \pm 0.08$ & $0.122 \pm 0.004$ & $0.862 \pm 0.034$ & $4.78 \times 10^{-20} \pm 5.13 \times 10^{-12}$ \\
\hline 436 & $15.1 \pm 0.2$ & $1.50 \pm 0.06$ & $2.97 \pm 0.04$ & $0.131 \pm 0.002$ & $0.680 \pm 0.014$ & $6.30 \times 10^{-14} \pm 1.34 \times 10^{-16}$ \\
\hline 965 & $15.1 \pm 0.2$ & $1.50 \pm 0.06$ & $9.03 \pm 0.08$ & $0.139 \pm 0.002$ & $0.290 \pm 0.006$ & $7.27 \times 10^{-8} \pm 3.82 \times 10^{-9}$ \\
\hline
\end{tabular}

Table S3 Structural parameters obtained from the analysis of the SANS data of different concentrations of $d-C_{12} T A B$ in h-ChBr:h-Glyc.

\begin{tabular}{|c|c|c|c|c|c|c|}
\hline$\left[\mathrm{C}_{12} \mathrm{TAB}\right] / \mathrm{mM}$ & $\mathrm{r}_{\text {eq }} / \AA$ & $\mathrm{AR}$ & $\phi_{p} / \times 10^{-2}$ & $\mathrm{q}_{\mathrm{smax}} / \AA^{-1}$ & $\mathrm{~S}(0) / \mathrm{cm}^{-1}$ & $\Gamma_{\mathrm{k}}$ \\
\hline \multicolumn{7}{|c|}{$\mathrm{d}-\mathrm{C}_{12} \mathrm{TAB}$ in h-ChBr:h-Glyc } \\
\hline B1 & $14.5 \pm 0.2$ & $1.76 \pm 0.12$ & $1.08 \pm 0.14$ & $0.107 \pm 0.008$ & $0.844 \pm 0.034$ & $3.85 \times 10^{-20} \pm 6.42 \times 10^{-21}$ \\
\hline B2 & $14.5 \pm 0.2$ & $1.79 \pm 0.08$ & $7.37 \pm 0.06$ & $0.148 \pm 0.003$ & $0.388 \pm 0.008$ & $2.71 \times 10^{-9} \pm 1.91 \times 10^{-9}$ \\
\hline B3 & $14.5 \pm 0.2$ & $1.92 \pm 0.06$ & $17.3 \pm 0.2$ & $0.170 \pm 0.004$ & $0.141 \pm 0.003$ & $2.55 \times 10^{-6} \pm 2.55 \times 10^{-7}$ \\
\hline
\end{tabular}

\section{Excluded volume effects from a hard solvent shell}

An alternative theoretical model that could result in long-range interactions in DES is the presence of a strongly correlated solvent shell around the micelle. In order to validate this model, the experimental S(q) of $\mathrm{C}_{12}$ TAC in d-ChCl:d-Glyc and in $\mathrm{D}_{2} \mathrm{O}$ were fitted to an effective $\mathrm{HS}$ model, as presented in the main text. The results from this analysis are presented in Figure S3 and Table S4. 


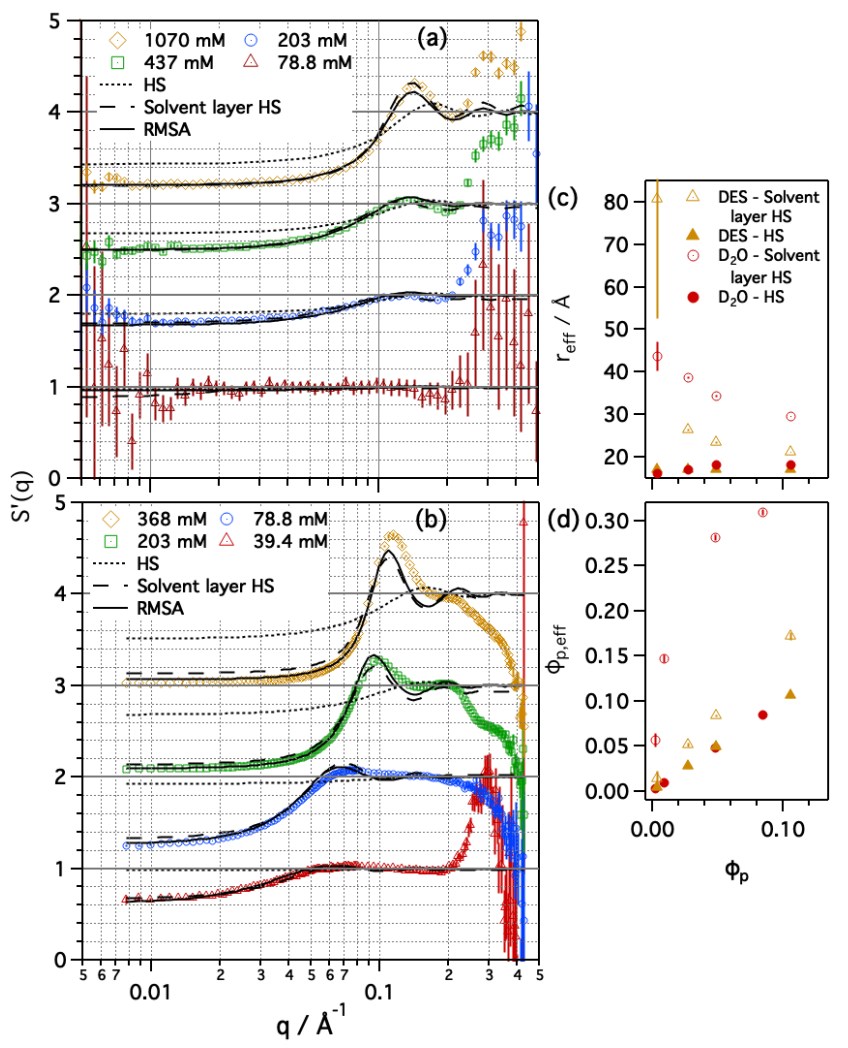

Figure S3 S(q)' data and best fits from different concentrations of $h-C_{12} T A C$ micelles in (a) 1:2 d-ChCl:d-Glyc and (b) $D_{2} O$. The concentrations of surfactant are presented in the legend of graph (a) and (b). Data were modelled using the HS model (dotted lines), HS model including a solvent layer around the particle (dashed lines) and RMSA model (solid lines). Data and models were scaled for clarity. Variation of (c) $r_{\text {eff }}$ and $\varphi_{p, \text { eff }}$ as a function of micelle volume fraction in DES and $D_{2} \mathrm{O}$ as shown in the legend of graph (c). Where not seen, the error bars are within the markers.

Table S4 Results from the analysis of the data of different concentrations of $\mathrm{d}-\mathrm{C}_{12} \mathrm{TAB}$ in $\mathrm{h}-\mathrm{ChBr}$ : $\mathrm{h}-\mathrm{Glyc}$ using a HS model that includes a solvent layer around the particle. The parameters derived from the analysis of the scattering data using the RMSA approach are included for comparison.

\begin{tabular}{|c|c|c|c|c|c|c|}
\hline$[\mathrm{C} 12 \mathrm{TAC}] / \mathrm{mM}$ & $\mathrm{r}_{\text {eff }} / \AA$ & $\phi_{\mathrm{p}, \text { eff }} / \times 10^{-2}$ & $\mathrm{r}_{\text {eff, P(q) }} / \AA$ & $\phi_{\mathrm{p}, \mathrm{P}(\mathrm{q})} / \times 10^{-2}$ & $\Delta \mathrm{r}_{\text {eff }} / \%$ & $\Delta \phi_{\mathrm{p}} / \%$ \\
\hline 78.8 & $80.7 \pm 28.1$ & $1.36 \pm 0.84$ & $17.1 \pm 0.3$ & $0.35 \pm 0.03$ & $372 \pm 130$ & $280 \pm 190$ \\
\hline 203 & $26.5 \pm 0.2$ & $5.16 \pm 0.17$ & $17.1 \pm 0.3$ & $2.79 \pm 0.04$ & $49 \pm 3$ & $84 \pm 3$ \\
\hline 437 & $23.4 \pm 0.2$ & $8.35 \pm 0.09$ & $17.1 \pm 0.3$ & $4.87 \pm 0.05$ & $37 \pm 3$ & $71 \pm 2$ \\
\hline 1070 & $21.1 \pm 0.1$ & $17.2 \pm 0.4$ & $17.1 \pm 0.2$ & $10.6 \pm 0.1$ & $23 \pm 1$ & $61 \pm 2$ \\
\hline \multicolumn{7}{|c|}{$\mathrm{D}_{2} \mathrm{O}$} \\
\hline 39.4 & $43.8 \pm 3.5$ & $5.63 \pm 0.71$ & $16.1 \pm 0.4$ & $0.23 \pm 0.03$ & $173 \pm 14$ & $2300 \pm 100$ \\
\hline 78.8 & $38.6 \pm 0.3$ & $14.7 \pm 0.4$ & $17.1 \pm 0.3$ & $0.93 \pm 0.03$ & $155 \pm 5$ & $1500 \pm 100$ \\
\hline 203 & $34.4 \pm 0.2$ & $28.1 \pm 0.3$ & $18.1 \pm 0.3$ & $4.82 \pm 0.02$ & $90 \pm 3$ & $480 \pm 10$ \\
\hline 368 & $29.6 \pm 0.1$ & $30.9 \pm 0.3$ & $18.3 \pm 0.2$ & $8.49 \pm 0.02$ & $61 \pm 1$ & $260 \pm 10$ \\
\hline
\end{tabular}

\section{Monomer concentration of counterion exchanged surfactants in DES}

As the fitted micelle volume fractions in DES are lower than the total surfactant volume fraction, it is expected that a certain amount of solubilized surfactant monomer remains in solution. Using the total surfactant volume fraction $\left(\phi_{s}\right)$, as calculated from the surfactant concentration in the sample, and the fitted micelle volume fraction $\left(\phi_{p}\right)$, the volume fraction and concentration of surfactant in the monomeric form $\left(\phi_{m}\right.$ and $\left[\mathrm{C}_{12} \mathrm{TA}^{+}\right]_{\mathrm{m}}$, respectively) were calculated for each surfactant concentration. The density of the counterionexchanged surfactants was approximated as $1 \mathrm{~g} / \mathrm{cm}^{3}$ for the calculations. The results from the calculations are presented in Table S5. 
Table S5 Calculated monomer volume fraction and concentration of monomers for different surfactant concentrations of $\mathrm{h}-\mathrm{C}_{12} \mathrm{TA}^{+}$in 1:2 d-ChCl:d-Glyc: $\mathrm{SO}_{4}{ }^{-2}, \mathrm{Br}^{-}, \mathrm{NO}_{3}{ }^{-}$, and $\mathrm{Cl}^{-}$.

\begin{tabular}{|c|c|c|c|c|}
\hline$\left[\mathrm{C}_{12} \mathrm{TA}^{+}\right] / \mathrm{mM}$ & $\phi_{s} / \times 10^{-2}$ & $\phi_{p} / \times 10^{-2}$ & $\phi_{m} / \times 10^{-2}$ & {$\left[\mathrm{C}_{12} \mathrm{TA}^{+}\right]_{\mathrm{m}} / \mathrm{mM}$} \\
\hline \multicolumn{5}{|c|}{$\mathrm{h}-\mathrm{C}_{12} \mathrm{TAC}$ in d-ChCl:d-Glyc } \\
\hline 78.8 & 2.04 & $0.35 \pm 0.03$ & $1.69 \pm 0.03$ & $57.3 \pm 1.0$ \\
\hline 203 & 5.09 & $2.79 \pm 0.04$ & $2.30 \pm 0.04$ & $78.3 \pm 1.4$ \\
\hline 437 & 10.3 & $4.87 \pm 0.05$ & $5.47 \pm 0.05$ & $186 \pm 2$ \\
\hline 1070 & 22.0 & $10.6 \pm 0.1$ & $11.4 \pm 0.1$ & $387 \pm 4$ \\
\hline \multicolumn{5}{|c|}{$\mathrm{h}-\mathrm{C}_{12} \mathrm{TAB}$ in d-ChCl:d-Glyc } \\
\hline 79.7 & 2.43 & $1.08 \pm 0.03$ & $1.34 \pm 0.03$ & $45.7 \pm 1.0$ \\
\hline 205 & 5.60 & $3.60 \pm 0.05$ & $2.00 \pm 0.05$ & $68.2 \pm 1.7$ \\
\hline 422 & 11.4 & $7.01 \pm 0.08$ & $4.39 \pm 0.08$ & $149 \pm 3$ \\
\hline 943 & 23.4 & $12.6 \pm 0.1$ & $10.8 \pm 0.1$ & $367 \pm 3$ \\
\hline \multicolumn{5}{|c|}{$\mathrm{h}-\mathrm{C}_{12} \mathrm{TANO}_{3}$ in d-ChCl:d-Glyc } \\
\hline 83.9 & 2.38 & $0.90 \pm 0.05$ & $1.48 \pm 0.05$ & $50.3 \pm 0.7$ \\
\hline 200 & 5.50 & $2.87 \pm 0.04$ & $2.63 \pm 0.04$ & $89.6 \pm 1.4$ \\
\hline 426 & 11.0 & $5.37 \pm 0.05$ & $5.64 \pm 0.05$ & $192 \pm 2$ \\
\hline 932 & 21.3 & $8.22 \pm 0.06$ & $13.1 \pm 0.1$ & $445 \pm 3$ \\
\hline \multicolumn{5}{|c|}{$\mathrm{h}-\mathrm{C}_{12} \mathrm{TA}\left(\mathrm{SO}_{4}\right)_{1 / 2}$ in d-ChCl:d-Glyc } \\
\hline 86.0 & 2.32 & $0.29 \pm 0.07$ & $2.03 \pm 0.07$ & $69.1 \pm 1.4$ \\
\hline 205 & 5.36 & $1.13 \pm 0.08$ & $4.23 \pm 0.08$ & $144 \pm 3$ \\
\hline 436 & 10.8 & $2.97 \pm 0.04$ & $8.07 \pm 0.04$ & $275 \pm 2$ \\
\hline 965 & 21.1 & $9.03 \pm 0.08$ & $12.1 \pm 0.1$ & $411 \pm 3$ \\
\hline
\end{tabular}

\section{Modelling approach for determining Coulomb coupling constants}

In order to directly compare the coupling constant for a given surfactant concentration, the experimental results for the coupling constant were modelled using an empirical approach for each of the studied surfactants. The Coulomb coupling constant versus the micelle volume fraction were fitted to the following equation:

$$
\log \left(\Gamma_{k}\right)=a+b \log \left(\phi_{p}\right)
$$

Subsequently, the Coulomb coupling constant of each system was evaluated for an arbitrary micelle volume fraction $\left(\phi_{p}\right)$. The results from the modelling are included in Figure S3. The models and calculations are presented in Table S4.
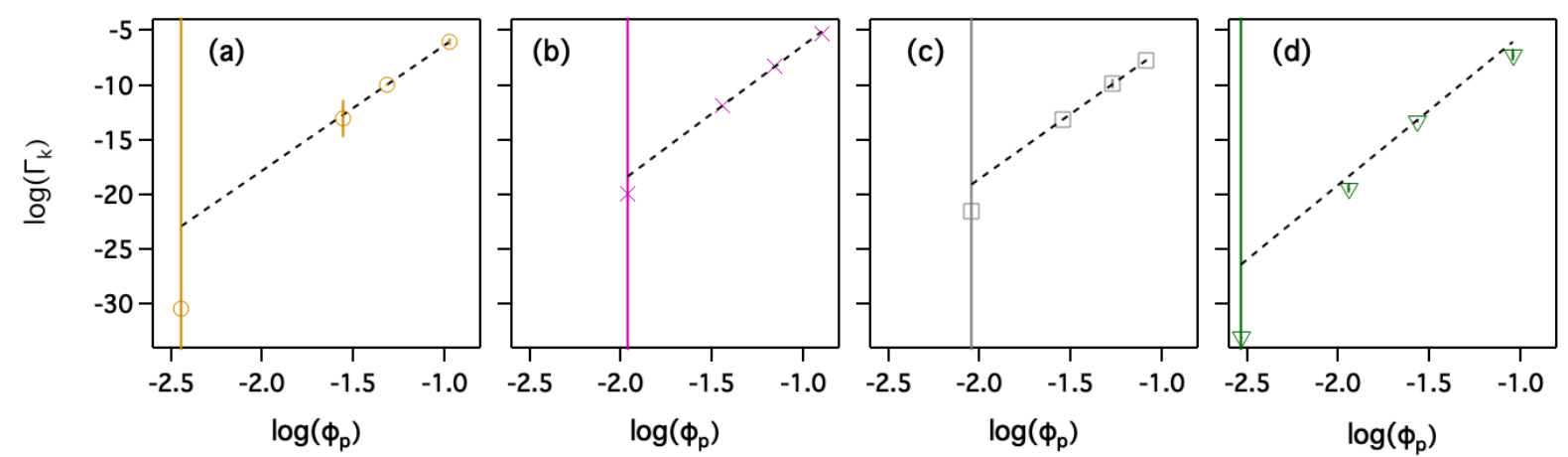

Figure S4 Modelling of the experimental Coulomb coupling constants for (a) h- $C_{12} T A C,(b) h-C_{12} T A B,(c) h-C_{12} T_{A N O}$, and (d) $\mathrm{h}-\mathrm{C}_{12} \mathrm{TA}\left(\mathrm{SO}_{4}\right)_{1 / 2}$ micelles in 1:2 d-ChCl:d-Glyc as a function of surfactant volume fraction. The models are presented as black dashed lines. Where not seen, the error bars are within the markers.

Table S6 Results from the modelling presented in Figure S3 using Equation S1. The Coulomb coupling constant was calculated for a volume fraction of micelles of 0.1 for comparison.

\begin{tabular}{|c|c|c|c|}
\hline System & $\mathrm{a}$ & $\mathrm{b}$ & $\Gamma_{\mathrm{k}}\left(\phi_{p}=0.1\right)$ \\
\hline $\mathrm{h}-\mathrm{C}_{12} \mathrm{TAC}$ in d-ChCl:d-Glyc & $5.598 \pm 0.129$ & $11.913 \pm 0.135$ & $4.841 \times 10^{-7} \pm 9.626 \times 10^{-9}$ \\
\hline h- $\mathrm{C}_{12} \mathrm{TAB}^{-1}$ in d-ChCl:d-Glyc & $5.430 \pm 0.109$ & $11.905 \pm 0.091$ & $3.348 \times 10^{-7} \pm 6.714 \times 10^{-9}$ \\
\hline h- $\mathrm{C}_{12} \mathrm{TANO}_{3}$ in d-ChCl:d-Glyc & $5.183 \pm 0.086$ & $11.856 \pm 0.117$ & $2.123 \times 10^{-7} \pm 3.185 \times 10^{-9}$ \\
\hline h-C $\mathrm{C}_{12} \mathrm{TA}^{-}\left(\mathrm{SO}_{4}\right)_{1 / 2}$ in d-ChCl:d-Glyc & $7.123 \pm 0.420$ & $13.385 \pm 0.324$ & $5.467 \times 10^{-7} \pm 1.219 \times 10^{-8}$ \\
\hline
\end{tabular}




\section{Variation of the Coulomb coupling constant with the solvent ionic strength}

As seen in the main text, the strength of long-range interactions in DES is significantly reduced compared to those in water. This difference was attributed to the higher ionic strength of the solvent. Using Equation 5, theoretical calculations of the coupling constant were performed for a model particle with $r_{\text {eff }}=18.35 \AA$, $\mathrm{AR}=1.3, \mathrm{z}_{\mathrm{p}}=40$ and $\phi_{\mathrm{p}}=0.085$ in $\mathrm{D}_{2} \mathrm{O}\left(\varepsilon=77.9,30^{\circ} \mathrm{C}\right)$. The coupling constant was determined for different solvent ionic strengths: $10,25,50,100,250$ and $500 \mathrm{mM}$. Using the values of the Coulomb coupling constant, the theoretical scattering for the particles was determined using a uniform ellipsoid form factor and the RMSA model. The results from the calculations are presented in Table S7 and Figure S5. The theoretical scattering for the same particles interacting through excluded volume effects (HS) is presented for comparison.

Table S7. Theoretical predictions of the Coulomb coupling constant at different ionics strengths. The calculations were performed for a model particle with $r_{\text {eff }}=18.35 \AA, A R=1.3, z_{p}=40$ and $\phi_{p}=0.085$ in $D_{2} O\left(\varepsilon=77.9,30^{\circ} \mathrm{C}\right)$. The value for hard sphere interactions is included for comparison.

\begin{tabular}{|c|c|}
\hline $1 / \mathrm{mM}$ & $\Gamma_{\mathrm{k}}$ \\
\hline 10 & $5.27 \mathrm{E}+02$ \\
\hline 25 & $5.90 \mathrm{E}+01$ \\
\hline 50 & $5.40 \mathrm{E}+00$ \\
\hline 100 & $2.01 \mathrm{E}-01$ \\
\hline 250 & $3.57 \mathrm{E}-04$ \\
\hline 500 & $3.40 \mathrm{E}-07$ \\
\hline HS & 0 \\
\hline
\end{tabular}
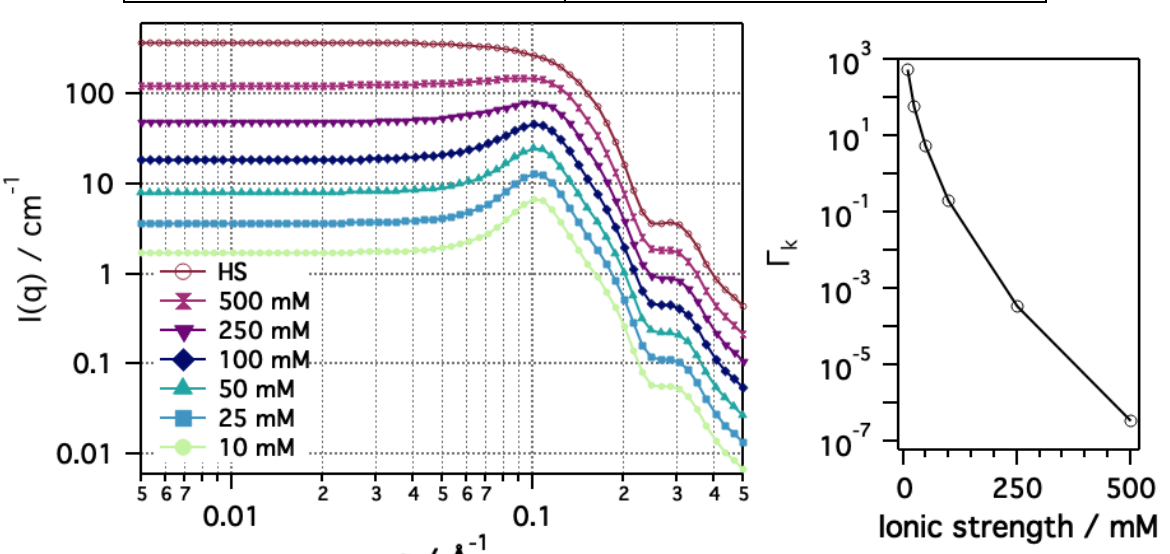

$q / \AA^{-1}$

Figure S5. Calculated scattering intensities for the model particle interacting electrostatically through different the Coulomb coupling constants (see Table S5).

\section{Sensitivity analysis of the correlation between the solvent ionic strength and particle charge}

As introduced in the main text, the coupling between the solvent ionic strength and particle surface charge in the calculation of the Coulomb coupling constant limits the accuracy of the determination of these individual variables. To study this correlation, a sensitivity analysis was performed. Using the experimental data from $1070 \mathrm{mM} \mathrm{C}_{12} \mathrm{TAC}$ in 1:2 d-ChCl:d-Glyc, the pairs of particle charge and solvent ionic strength were determined for the Coulomb coupling constant that fits the data. Initially, the aggregation number ( $\mathrm{N}_{\mathrm{agg}}$ ) of the micelles was calculated using the equation:

$$
N_{\text {agg }}=\frac{V_{p}}{V_{m}}
$$

$V_{p}$ is the volume of the micelle and $V_{m}$ is the volume of the surfactant monomer. The $V_{m}$ was calculated using Tanford equations and is $350.2 \AA^{3}$ (Tanford, J. Phys. Chem. 1972). The particle charge values were obtained considering a realistic upper and lower boundary for the counterion dissociation, i.e. $\alpha_{\min }=20 \%$ and $\alpha_{\text {max }}=80 \%$, where $z_{p}=a N_{\text {agg. }}$. The model was parametrized using and $\varepsilon=22.8$, and $r_{\text {eff }}=17.1 \AA$ and $N_{\text {agg }}=52 \pm 3$ from the structural analysis of the micelles. Using these values in the analysis, the experimental data were fitted to obtain the Coulomb coupling constant, particle surface potential, and the effective ionic strength of the solvent. The results from the sensitivity analysis are presented in Table S8. 
Table S8 Results from the sensitivity analysis of the correlation between the particle charge and solvent ionic strength.

\begin{tabular}{|c|c|c|c|c|c|}
\hline$\alpha$ & $z_{p}$ & $\psi_{0} / \times 10^{-2} \mathrm{~V}$ & $\mathrm{I} / \mathrm{mM}$ & $\Gamma_{\mathrm{k}} / \times 10^{-7}$ & $\chi^{2}$ \\
\hline 0.2 & 10.4 & $3.79 \pm 0.03$ & $338 \pm 6$ & $8.86 \pm 0.05$ & 28.123 \\
\hline 0.3 & 15.6 & $5.67 \pm 0.04$ & $398 \pm 5$ & $8.80 \pm 0.05$ & 24.036 \\
\hline 0.4 & 20.8 & $7.18 \pm 0.05$ & $435 \pm 6$ & $8.88 \pm 0.06$ & 21.394 \\
\hline 0.5 & 26.0 & $8.64 \pm 0.05$ & $465 \pm 7$ & $8.87 \pm 0.06$ & 19.074 \\
\hline 0.6 & 31.2 & $10.1 \pm 0.1$ & $491 \pm 5$ & $8.86 \pm 0.04$ & 18.256 \\
\hline 0.7 & 36.4 & $11.5 \pm 0.1$ & $513 \pm 6$ & $8.84 \pm 0.05$ & 19.514 \\
\hline 0.8 & 41.6 & $13.1 \pm 0.1$ & $538 \pm 6$ & $8.82 \pm 0.06$ & 20.797 \\
\hline
\end{tabular}

The analysis shows that the correlation between the surface potential and ionic strength results in pairs of values that give fits of relatively equal quality $\left(\chi^{2}\right.$ between 20 and 30). These pairs of values result in the same Coulomb coupling constant, as the contact potential for the interparticle repulsion is determined from the experimental scattering. The difference in the quality of the fits is marginal due to the correlation between the two parameters. However, it is seen that the effective ionic strength of the solvent is around 400-500 $\mathrm{mM}$.

\section{References}

1. Sanchez-Fernandez, A.; Hammond, O. S.; Edler, K. J.; Arnold, T.; Doutch, J.; Dalgliesh, R. M.; Li, P.; Ma, K.; Jackson, A. J., Counterion binding alters surfactant self-assembly in deep eutectic solvents. Phys. Chem. Chem. Phys. 2018, 20 (20), 13952-13961. 